\title{
Correlation Between Light Levels and the Development of Deprivation Myopia
}

\author{
Cindy Karouta ${ }^{1}$ and Regan Scott Ashby ${ }^{1,2}$ \\ ${ }^{1}$ Centre for Research in Therapeutic Solutions, Biomedical Sciences, Faculty of Education, Science, Technology and Mathematics, \\ University of Canberra, Australian Capital Territory, Australia \\ ${ }^{2}$ Research School of Biology, Australian National University, Canberra, Australian Capital Territory, Australia
}

Correspondence: Regan S. Ashby, Centre for Research in Therapeutic Solutions, Faculty of Education, Science, Technology and Mathematics, University of Canberra, University Drive, Bruce, Canberra, Australia; regan.ashby@canberra.edu.au.

Submitted: August 18, 2014 Accepted: November 24, 2014

Citation: Karouta C, Ashby RS. Correlation between light levels and the development of deprivation myopia. Invest Ophthalmol Vis Sci. 2015;56:299-309. DOI:10.1167/ iovs.14-15499

\begin{abstract}
Purpose. In chicks, daily exposure to bright light (15,000 lux) retards the development of form-deprivation myopia (FDM) by roughly $60 \%$. This study investigated whether higher light intensities increase the amount of protection against FDM, and whether protection and light intensity are correlated. Furthermore, we examined if exposure to bright light can prevent the progression of FDM or whether it affects only the onset of experimental myopia.

Methods. Experiment 1: Chicks wore translucent diffusers monocularly for a period of 7 days, with exposure to one of five light intensities $(500,10,000,20,000,30,000$, and 40,000 lux, $n$ $=12$ per group). Experiment 2: Chickens wore translucent diffusers monocularly for 11 days and were split into three groups: (1) chicks reared under 500 lux, (2) chicks reared under 40,000 lux, and (3) chicks reared under 500 lux for the first 4 days and 40,000 lux for the remaining 7 days.

Results. A significant correlation was observed between $\log$ light intensity and the development of FDM, with a lesser myopic refraction $(F(28,330)=60.86, P<0.0001)$ and shorter axial length $(F(4,20)=8.87, P<0.0001)$ seen with increasing light intensities. The progression of FDM was halted in chicks that were switched from 500 to 40,000 lux.

Conclusions. The level of protection from the development of FDM increases with increasing light intensity. Daily exposure to 40,000 lux almost completely prevents the onset of FDM and, once myopia is established, halts further progression.
\end{abstract}

Keywords: myopia, eye growth, outdoor activity, light intensity
$\mathrm{T}$ he visual disorder myopia (short-sightedness) is due to a mismatch between the optical power of the eye and its axial length. This is due most commonly to excessive elongation of the eye during development. A rapid increase in the prevalence of myopia has been observed, most notably in urban East Asia. ${ }^{1-6}$ A number of epidemiological studies have reported a strong negative correlation between time spent outdoors and the development of myopia, with those children who spend greater amounts of time outdoors less likely to become myopic. ${ }^{7-13}$ Rose et al. ${ }^{10}$ postulated that this protective effect may be driven by light-stimulated dopamine release from the retina, associated with the higher illumination levels experienced outdoors. In support of this hypothesis, animal studies have shown that the development of experimental myopia can be retarded by daily exposure to bright light (15,000-30,000 lux), relative to that seen under normal laboratory lighting levels (500 lux). ${ }^{14-17}$ In chicks, this protective effect can be abolished by the daily administration of a dopamine D2 receptor antagonist, spiperone. ${ }^{15}$ This suggests that in chicks, the protective effects of bright light are driven, to some extent, by retinal dopamine.

In chickens, exposure to 15,000 lux, for a period of 5 hours per day, retards the development of form-deprivation myopia (FDM) by roughly $60 \%$ relative to the refractive changes seen in chicks fitted with diffusers and reared under normal laboratory lighting levels (500 lux). ${ }^{14}$ This study examines whether greater protection is afforded with higher light intensities (ranging from
$10,000-40,000$ lux). We postulate that a positive correlation exists between light levels and the degree to which the development of FDM is prevented. In support of this hypothesis, Ashby et al. ${ }^{14}$ have shown that the extent to which brief periods of normal vision retard the development of $\mathrm{FDM}^{18,19}$ is proportional to the light levels experienced during diffuser removal. ${ }^{14}$ Further, Cohen et al. ${ }^{20}$ have reported that the normal emmetropization process in chicks appears to depend on light intensity. The authors observed that a more hyperopic refraction is maintained over a 90-day period when chicks are reared under higher luminance levels (10,000 lux). In contrast, rearing chicks under low light (50 lux) induces a myopic shift by 90 days $(-2.41$ diopters [D]). Together, this work suggests that a correlation exists between luminance levels and refractive development. The present study also examines whether bright light can prevent further progression in already myopic eyes, or whether it is effective only at retarding the onset of experimental myopia. This is an important question, as epidemiological studies have suggested that increased time outdoors can reduce the probability of myopia onset but does not appear to affect the rate of progression. ${ }^{12,13}$

\section{MeTHODS}

\section{Animal Housing}

One-day-old male White Cockerel chickens were obtained from Barter \& Sons Hatchery (Luddenham, NSW, Australia). 
Chickens were housed in temperature-controlled rooms under a 12:12 hour light:dark cycle, with lights on at $7 \mathrm{AM}$ and off at 7 PM. Chickens had access to unlimited amounts of food and water, and were given 4 days to become accustomed to their environment before experiments started. Authorization to conduct experiments using animals was approved by the University of Canberra Animal Ethics Committee under the ACT Animal Welfare Act 1992 (project number CEAE 13-03) and conformed to the ARVO Statement for the Use of Animals in Ophthalmic and Vision Research.

\section{Experimental Design}

Experiment 1A: Chickens were monocularly treated with translucent diffusers for 7 days and reared under a normal 12:12 hour light:dark cycle. During the light phase, chicks were exposed to either normal laboratory light levels (500 lux, $n=12)$ or to one of four higher illumination levels $(10,000$, 20,000, 30,000, and 40,000 lux, $n=12$ per light intensity). Chicks in the high light groups (10,000-40,000 lux) were exposed to these intensities for a period of 6 hours per day (11 AM-5 PM), over the 7 days, while being reared under normal laboratory lighting levels for the remainder of the light period (7 AM-11 AM and 5 PM-7 PM). Axial length was measured on the day prior to the commencement of the experiment and on day 6. Refraction was measured daily at 9 AM.

Experiment 1B: To determine if light intensity affects normal ocular development, age-matched untreated control birds were exposed to either normal laboratory light levels (500 lux, $n=12$ ) or to one of four higher illumination levels $(10,000,20,000,30,000$, and 40,000 lux, $n=12$ per light intensity) for a period of 7 days. As with experiment $1 \mathrm{~A}$, chicks in the high light groups $(10,000-40,000$ lux $)$ were exposed to these intensities for a period of 6 hours per day (11 AM-5 PM), over the 7 days, while being reared under normal laboratory lighting levels for the remainder of the light period. Axial length was measured on the day prior to the commencement of the experiment and on day 6. Refraction was measured daily at $9 \mathrm{AM}$.

Experiment 2: To investigate if the progression of FDM could be prevented by bright light exposure, chickens monocularly wore translucent diffusers for 11 days and were split into three treatment groups: (1) chicks reared under 500 lux for the entirety of the light phase, (2) chicks exposed to 40,000 lux, for a period of 6 hours per day, while being reared under 500 lux for the remainder of the light phase, and (3) chicks initially reared under 500 lux for a period of 4 days to induce deprivation myopia. Following this, chicks were switched to daily exposure to 40,000 lux, for a 6-hour period per day, for the remaining 7 days. Axial length was measured on the day prior to the commencement of the experiment and on day 10. Refraction was measured daily at 9 AM.

For all experiments, on the day prior to treatment, following ultrasound measurements, Velcro mounts were fitted around the left eye with Loctite super glue (Henkel, Düsseldorf, Germany). On the following day, the translucent diffusers, fitted to matching Velcro rings, were placed onto the mounts.

\section{Measurement of Ocular Parameters}

Refraction and axial length were measured by automated infrared photoretinoscopy (system provided by Frank Schaeffel, University of Tuebingen, Germany) and A-scan ultrasonography (Biometer AL-100; Tomey Corporation, Nagoya, Japan), respectively. ${ }^{14,15}$

\section{Light Sources}

Illumination of the cages was produced by a custom-made system composed of 10 banks of LED (light-emitting diode) lamps, with an illumination angle of $60^{\circ}$ for each individual LED (Electronics Department, Research School of Biology, Australian National University). The illumination output was adjustable by a dimmer system on a linear scale between 0 and 45,000 lux. Each LED bank was composed of an equal mix of cool (400-650 nm, peaking at $450 \mathrm{~nm}$ ) and warm (430-700 $\mathrm{nm}$, peaking at $630 \mathrm{~nm}$ ) LED modules, generating minimal to no heat load, even at maximum intensity. The final spectral composition was similar to that produced by quartz-halogen bulbs, which were previously used to generate high light levels. ${ }^{14,15}$ The lighting system did not emit in either the infrared (IR) or ultraviolet (UV) spectrum. Illumination levels were uniform across the cage area $(1.6 \times 0.8$ meters $)$, as measured by an IL-1700 Research Radiometer (International Light, Inc., Chula Vista, CA, USA), with the lighting system sitting 1.5 meters above the cage roof. Cages were placed against a single wall, allowing a viewing distance of 5 meters in three directions. All reported luminances used in this study were measured at the level of the cage floor, with lighting levels continuously monitored within the cages by an automated system using $\mathrm{HOBO}$ data loggers (Onset HOBO Data Loggers, Bourne, MA, USA).

\section{Histology}

To determine if bright light exposure induces neuronal damage, retinal tissue from chicks reared under 40,000 and 500 lux, for a period of 7 days, was processed for histology. Following deep anesthesia (5\% isoflurane in oxygen), chicks were euthanized and the eyes rapidly enucleated. Eyes were hemisected, with the anterior portion and vitreous body discarded, while the posterior eye cup was fixed by immersion in $4 \%$ paraformaldehyde in $0.1 \mathrm{M}$ phosphate-buffered saline (PBS, pH 7.4) for 4 hours at $4^{\circ} \mathrm{C}$. Eye cups were then left overnight in a $30 \%$ sucrose solution, dissolved in $0.1 \mathrm{M}$ PBS, for cryoprotection, before being embedded in optimal cutting temperature (OCT) compound (Tissue-Tek; Thermo Fischer Scientific, Scoresby, VIC, Australia). Retinal cyrosections $8 \mu \mathrm{m}$ thick were cut from the nasotemporal plane using a cryostat (CM1850; Leica Microsystems, North Ryde, NSW, Australia). Sections were mounted onto gelatin-coated glass slides and airdried overnight. Sections were stained for morphological examination using hematoxylin and eosin (H\&E; Thermo Fischer Scientific) or TUNEL labeled for analysis of cell death following the protocol of Maslim et al. ${ }^{21}$ For TUNEL labeling, slides were immersed in $70 \%$ ethanol for 30 minutes before being washed in distilled water. Peroxidase activity was blocked by incubation in $3 \% \mathrm{H}_{2} \mathrm{O}_{2}$ for 10 minutes. Slides were then incubated in terminal deoxynucleotide transferase buffer ( $3 \mathrm{mM}$ Trizma base, $14 \mathrm{mM}$ sodium cacodylate, and $0.11 \mathrm{mM}$ cobalt chloride) for 10 minutes before incubation in a reaction buffer containing terminal deoxynucleotide transferase (TdT, 0.03 units $/ \mu \mathrm{L}$; Thermo Fischer Scientific), biotinylated deoxyuridine triphosphate (biotin-16-dUTP, $4 \mu \mathrm{M}$; Roche, Castle Hill, NSW, Australia) in a TdT buffer for 1 hour at $37^{\circ} \mathrm{C}$. The reaction was stopped by incubation in a $300 \mathrm{mM} \mathrm{NaCl}$ buffer containing $30 \mathrm{mM}$ sodium citrate. Nonspecific binding was blocked by incubation in a $1 \%$ bovine serum albumin solution for 15 minutes. Secondary labeling was achieved through incubation with streptavidin-conjugated Alexa Fluor 594 (Life Technologies, Mulgrave, VIC, Australia) for 1 hour at room temperature.

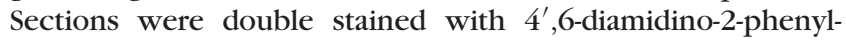
indole (DAPI; Life Technologies) DNA nuclear stain for visualization of cell layers. As a positive control, retinal tissue 


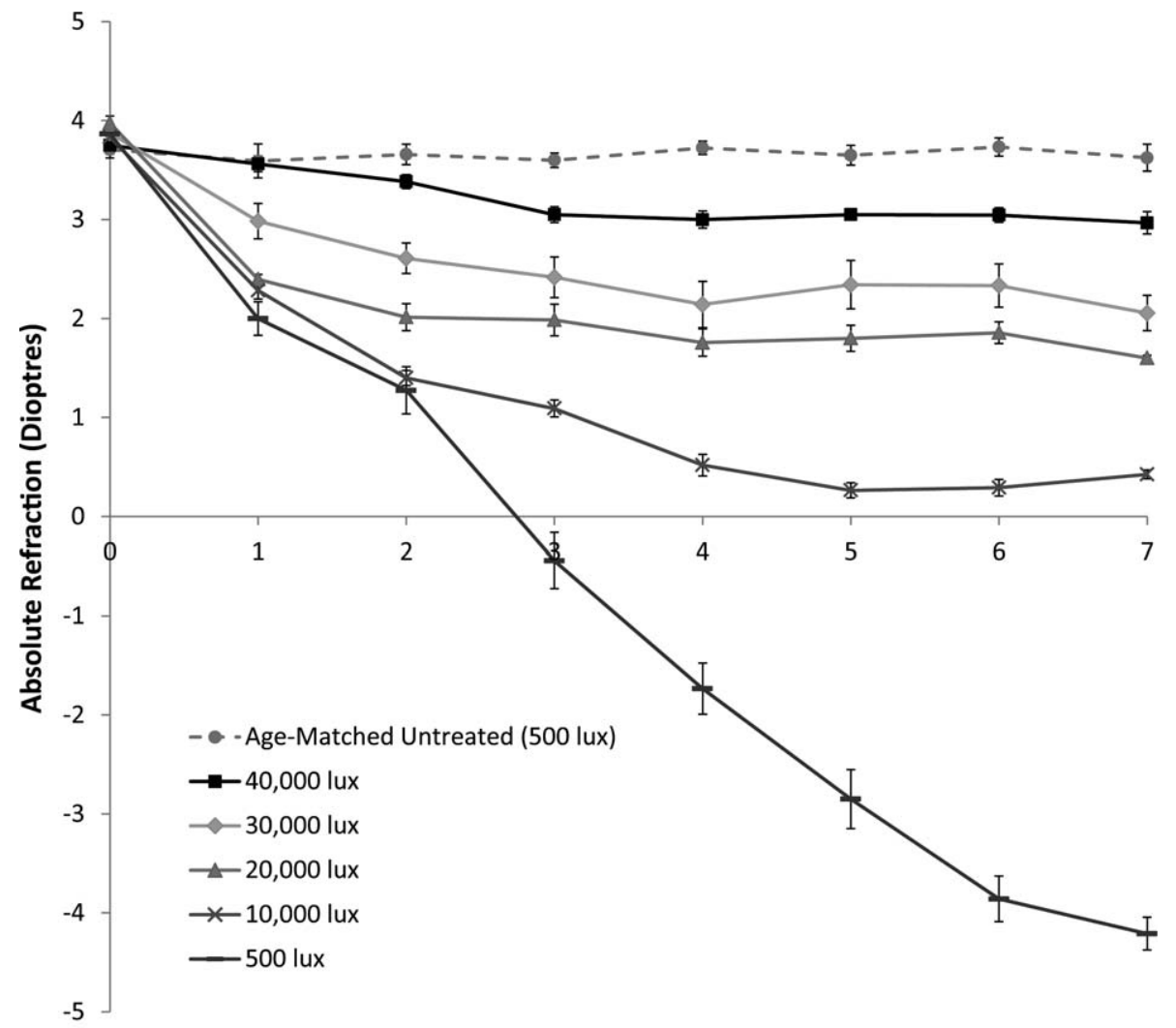

Time (days)

Figure 1. Absolute change in refraction over 8 days for diffuser-treated eyes from animals reared under one of five light intensities (500, 10,000, $20,000,30,000$, or 40,000 lux). The development of deprivation myopia was retarded proportionally to increasing light intensities. Plotted untreated control values represent the mean refraction, at each time point, for age-matched untreated control eyes. There was no statistical difference in refraction changes seen in contralateral control eyes or age-matched untreated control eyes in response to any light intensity. Therefore contralateral data are not plotted for ease of viewing. Error bars: standard error of the mean, $n=12$ per group.

from Sprague-Dawley (SD) rats, in which DNA damage and apoptosis had been induced through light damage, was processed simultaneously with the chicken retinal samples. To induce retinal damage, SD rats (90 days of age) were exposed to 1500 lux for a 24-hour period, which is known to induce photoreceptor apoptosis. ${ }^{22}$

\section{Statistics}

All results are presented as the mean \pm the standard error of the mean. For the analysis of changes in ocular parameters over time, a multivariate analysis of variance (MANOVA) with repeated measures design was used to compare the effect of light levels (between-subject variable) over time (withinsubject variable) on refraction development or axial length (dependent variables). Analysis of changes in ocular parameters between groups at specific treatment times was undertaken by post hoc testing using a Student's unpaired $t$-test, with Bonferroni correction for multiple testing. All statistical analyses were undertaken in SPSS (IBM, Armonk, NY, USA).

\section{RESULTS}

\section{Correlation Between Light Intensity and the} Development of Form-Deprivation Myopia Over Time

In Experiment 1A, light had a significant effect on refractive development in diffuser-treated eyes over the 8-day period measured $(F(28,330)=60.86, P<0.0001$; Fig. 1; Table 1$)$.

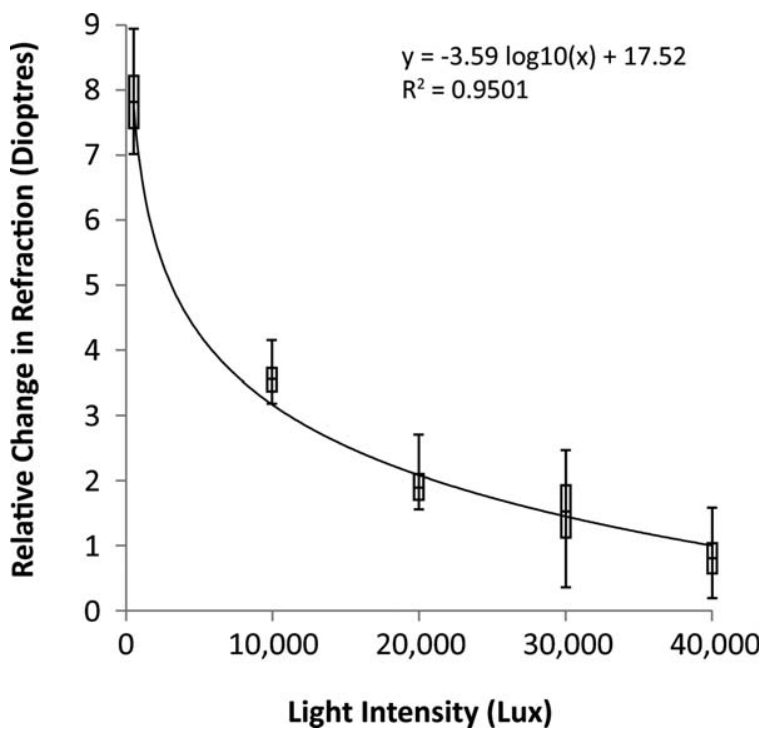

FIgURE 2. Plot of logarithmic regression of light intensity versus relative change in refraction over 8 days. Relative change in refraction represents the refractive value recorded for the diffuser-treated eye minus the value recorded for the contralateral control eye at each time point. The relative myopic change in refraction associated with diffuser wear regressed strongly with light intensity, which was best modeled by a logarithmic relationship. The percentage variation explained by the regression was $95.0 \%$. 
TABLE 1. Refractive Data From Diffuser-Treated Eyes Over 8 Days for All Light Intensities

\begin{tabular}{|c|c|c|c|c|c|c|c|c|c|}
\hline \multirow[b]{2}{*}{ Treatment } & \multirow[b]{2}{*}{ LI, Lux } & \multicolumn{8}{|c|}{ Refraction, Diopters } \\
\hline & & Day 0 & Day 1 & Day 2 & Day 3 & Day 4 & Day 5 & Day 6 & Day 7 \\
\hline $\begin{array}{l}\text { Consta } \\
\text { Contra }\end{array}$ & 00 & $3.75 \pm 0.09$ & $3.56 \pm 0.08$ & $3.38 \pm 0.07$ & $3.05 \pm 0.08$ & $3.00 \pm 0.09$ & $3.05 \pm 0.05$ & $3.05 \pm 0.07$ & 2.97 \\
\hline control & 40,000 & $3.75 \pm 0.09$ & $3.76 \pm 0.09$ & $3.76 \pm 0.09$ & $3.76 \pm 0.10$ & $3.82 \pm 0.08$ & $3.83 \pm 0.08$ & $3.86 \pm 0.09$ & $3.70 \pm 0.0$ \\
\hline $\begin{array}{l}\text { elative change } \\
\text { in refraction }\end{array}$ & & $0=$ & $-0.2 \pm 0.07$ & $-0.38 \pm$ & -0.7 & -0.8 & -0.7 & 0.13 & $-0 . / 3$ \\
\hline $\begin{array}{l}\text { Constant } \\
\text { Contrala }\end{array}$ & 30,000 & $3.88 \pm$ & $2.98 \pm 0.18$ & $2.61 \pm$ & $2.42 \pm$ & $2.14 \pm$ & $2.34 \pm 0.24$ & $2.33 \pm$ & $2.06 \pm$ \\
\hline $\begin{array}{l}\text { control } \\
\text { Relative change }\end{array}$ & 30,000 & $3.88 \pm 0.08$ & $3.84 \pm 0.07$ & $3.81 \pm 0.08$ & $3.87 \pm 0.09$ & $3.88 \pm 0.08$ & $3.88 \pm 0.09$ & $3.86 \pm 0.06$ & $3.80 \pm 0.0$ \\
\hline in refr & & $0.00 \pm 0.12$ & $-0.86 \pm 0.14$ & $-1.2 \pm 0.14$ & $-1.45 \pm 0.19$ & $-1.73 \pm 0.22$ & $-1.54 \pm 0.26$ & $-1.53 \pm 0.22$ & $-1.74 \pm 0.13$ \\
\hline $\begin{array}{l}\text { iffuser } \\
\text { al }\end{array}$ & 20,000 & $8 \pm 0$ & $2.40 \pm 0$ & & & & $1.80 \pm 0.13$ & $1.86 \pm$ & $1.60=$ \\
\hline $\begin{array}{r}\text { cor } \\
\text { Relat }\end{array}$ & 0,000 & $96 \pm 0.07$ & $3.79 \pm 0.10$ & $3.77 \pm 0.09$ & $3.69 \pm 0$ & $3.76 \pm 0.18$ & $3.70 \pm 0.17$ & $3.74 \pm 0.18$ & 373 \\
\hline $\begin{array}{l}\text { Relative } \\
\text { in ref }\end{array}$ & & $+0.02 \pm$ & $-1.39 \pm$ & -1 & -1 & -2.00 & -1 & -1 & -2.1 \\
\hline $\begin{array}{l}\text { Constant diffuser } \\
\text { Contralateral }\end{array}$ & 10,000 & $3.83 \pm$ & $2.28 \pm$ & 1.40 & 1.09 & $2 \pm$ & $0.26 \pm 0.08$ & $0.29 \pm$ & $0.43=$ \\
\hline $\begin{array}{l}\text { con } \\
\text { Relativ }\end{array}$ & 0,000 & $3.84 \pm 0.04$ & $3.83 \pm 0.04$ & $3.86 \pm 0.05$ & $3.85 \pm 0.04$ & $3.85 \pm 0.04$ & $3.85 \pm 0.04$ & $3.86 \pm 0.04$ & $3.87 \pm 0.04$ \\
\hline in $\mathrm{rc}$ & & $-0.01 \pm 0.06$ & $-1.54 \pm 0.09$ & $-2.46 \pm 0.09$ & -2 & -3 & -3 & \pm 0.09 & -3.44 \\
\hline $\begin{array}{l}\text { Constant diffuser } \\
\text { Contralateral }\end{array}$ & 500 & $3.87 \pm 0.07$ & $2.00 \pm 0.17$ & $1.28 \pm 0.24$ & $-0.44 \pm 0.28$ & $-1.73 \pm 0.26$ & $-2.85 \pm 0.30$ & $-3.86 \pm 0.23$ & $-4.21 \pm 0.17$ \\
\hline $\begin{array}{l}\text { control } \\
\text { elative chang }\end{array}$ & 500 & $3.87 \pm 0.07$ & $3.94 \pm 0.08$ & $3.95 \pm 0.09$ & $3.88 \pm 0.06$ & $3.94 \pm 0.08$ & $3.97 \pm 0.07$ & $3.88 \pm 0.06$ & $3.81 \pm 0.02$ \\
\hline in ref & & $0=$ & 0.19 & $-2.68=$ & -4.3 & $68 \pm 0.2$ & $-6.82 \pm 0$ & $-7.74 \pm 0.24$ & -8.0 \\
\hline
\end{tabular}

Relative change in refraction represents the refractive value recorded for the diffuser-treated eye minus the refractive value recorded for the contralateral control eye at each time point for each animal. Error, standard error of the mean; $n=12$ per group. LI, light intensity.

The amount of relative myopia that developed in diffusertreated eyes (refractive value in the diffuser-treated eye minus the refractive value in the contralateral eye) regressed strongly with light intensity and could be explained by a logarithmic relationship $\left(y=-3.59 \log 10(x)+17.52 ; r^{2}=0.95\right.$; Fig. 2$)$. The percentage of the variation explained by the regression was 95.0\%. Chickens reared under normal indoor lighting levels (500 lux) developed FDM to the greatest extent following 8 days of treatment (absolute refraction $-4.21 \pm 0.17 \mathrm{D}$, relative myopia $\Delta-8.02 \pm 0.21 \mathrm{D}$; Fig. 1 ; Table 1 ). In contrast, daily exposure to 40,000 lux, for a period of 6 hours per day, showed the least myopic shift in refraction following 8 days of treatment (absolute refraction $+2.97 \pm 0.11 \mathrm{D}$, relative myopia $\Delta-0.73 \pm 0.20$ D; Fig. 1; Table 1).

As with refraction, illumination levels had a significant negative correlation with the axial length of diffuser-treated eyes $(F(4,20)=8.87, P<0.0001$; Table 2$)$; the higher the light intensity, the shorter the length of the eye. Specifically, following 8 days of treatment, diffuser-treated chicks reared under 500 lux showed the longest eyes relative to the

TABLE 2. Axial Length Data From Diffuser-Treated Eyes Over 8 Days for All Light Intensities

\begin{tabular}{lrrr}
\hline & & & Axial Length, mm \\
\cline { 2 - 4 } \multicolumn{1}{c}{ Treatment } & LI, Lux & \multicolumn{1}{c}{ Day $\mathbf{~}$} & Day 6 \\
\hline Constant diffuser & 40,000 & $8.58 \pm 0.05$ & $8.77 \pm 0.03$ \\
Contralateral control & 40,000 & $8.62 \pm 0.08$ & $8.69 \pm 0.03$ \\
Relative change in axial length & & $-0.04 \pm 0.07$ & $+0.08 \pm 0.02$ \\
Constant diffuser & 30,000 & $8.53 \pm 0.03$ & $9.05 \pm 0.07$ \\
Contralateral control & 30,000 & $8.53 \pm 0.03$ & $8.74 \pm 0.03$ \\
Relative change in axial length & & $0.00 \pm 0.06$ & $+0.31 \pm 0.07$ \\
Constant diffuser & 20,000 & $8.57 \pm 0.03$ & $9.19 \pm 0.12$ \\
Contralateral control & 20,000 & $8.53 \pm 0.04$ & $8.79 \pm 0.11$ \\
Relative change in axial length & & $+0.04 \pm 0.10$ & $+0.40 \pm 0.19$ \\
Constant diffuser & 10,000 & $8.53 \pm 0.03$ & $9.26 \pm 0.11$ \\
Contralateral control & 10,000 & $8.57 \pm 0.05$ & $8.79 \pm 0.06$ \\
Relative change in axial length & & $-0.04 \pm 0.05$ & $+0.47 \pm 0.07$ \\
Constant diffuser & 500 & $8.53 \pm 0.02$ & $9.31 \pm 0.07$ \\
Contralateral control & 500 & $8.53 \pm 0.06$ & $8.67 \pm 0.04$ \\
Relative change in axial length & & $0.00 \pm 0.05$ & $+0.64 \pm 0.12$ \\
\hline$\quad$ Re & & \\
\hline
\end{tabular}

Relative change in axial length represents the axial length value recorded for the diffuser-treated eye minus the axial length value recorded for the contralateral control eye at each time point for each animal. Error, standard error of the mean; $n=12$ per group. 
contralateral control values (absolute axial length $9.31 \pm 0.07$ $\mathrm{mm}$, change in axial length relative to contralateral values $\Delta$ $+0.64 \pm 0.12 \mathrm{~mm}$; Table 2). The smallest change in axial length was seen in chicks reared under 40,000 lux (absolute axial length $8.77 \pm 0.03 \mathrm{~mm}$, change in axial length relative to contralateral values $\Delta+0.08 \pm 0.02 \mathrm{~mm}$; Table 2 ).

In Experiment 1B, light did not affect refractive development or axial length over the 8 days of treatment in agematched untreated control eyes (refraction, $F(28,246)=1.50$, $P=0.09$; axial length, $F(4,24)=1.02, P=0.15$; Tables 3,4$)$ or contralateral control eyes of diffuser-treated animals $(F$ (28, $294)=1.52, P=0.07 ; F(4,22)=1.12, P=0.09$, respectively). There was also no significant difference in the refractive development between these two control groups $(F(63,546)=$ 1.23, $P=0.06$ ). Light, therefore, did not modify refractive development or axial length in eyes without diffusers over the experimental period. Yoking between diffuser-treated and contralateral control eyes was not observed.

\section{Can Exposure to Bright Light Prevent the Progression of Deprivation Myopia?}

As in experiment 1 , chicks fitted with translucent diffusers and reared continuously under 500 lux in experiment 2 showed the largest myopic shift in refraction by day 11 (absolute refraction $-4.47 \pm 0.21 \mathrm{D}$, relative myopia $\Delta-8.39 \pm 0.20 \mathrm{D}$; Fig. 3; Table 5). In contrast, the development of FDM was again significantly retarded in chicks exposed to 40,000 lux for a period of 6 hours a day, compared to that seen under 500 lux or in the progression group (absolute refraction $+2.86 \pm 0.10$ $\mathrm{D}$, relative myopia $\Delta-0.83 \pm 0.12 \mathrm{D} ; F(20,180)=47.86, P<$ 0.0001 ; Fig. 3; Table 5). The progression group, as illustrated in Figure 3 and Table 5, showed a significant myopic shift in refraction over the initial 3 days of diffuser wear under 500 lux (absolute refraction $-1.17 \pm 0.21 \mathrm{D}$, relative myopia $\Delta-5.01$ $\pm 0.18 \mathrm{D} ; P<0.0001)$. Following transfer to 40,000 lux, these animals showed an initial hyperopic shift, relative to their contralateral control eyes, over the first 2 days (relative change in refraction $\Delta+1.20 \pm 0.28 \mathrm{D}$ ), before refractive changes stabilized (plateaued) around emmetropia for the following 5 days (Fig. 3; Table 5). Therefore, by day 11, chicks switched to 40,000 lux (FDM progression group) showed a less myopic refraction relative to that seen under 500 lux (relative myopia $\Delta-3.70 \pm 0.29$ vs. $\Delta-8.39 \pm 0.20 \mathrm{D}$, respectively; $P<0.0001$ ), but a more myopic refraction compared to those chicks exposed for the entire experimental period to 40,000 lux (relative myopia $\Delta-3.70 \pm 0.29$ vs. $\Delta$ $-0.83 \pm 0.12 \mathrm{D}$, respectively; $P<0.0001$ ).

Changes in axial length were proportional to the refractive changes seen. Chicks fitted with diffusers and reared continuously under 500 lux showed the longest eyes by day 10 (absolute axial length $9.54 \pm 0.13 \mathrm{~mm}$, change in axial length relative to contralateral values $\Delta+0.64 \pm 0.13 \mathrm{~mm}$; Table 6). Relative to the 500-lux group, chicks exposed to 40,000 lux had the shortest eyes among diffuser-treated animals (change in axial length relative to contralateral values $\Delta+0.64 \pm 0.13$ vs. $\Delta+0.17 \pm 0.10 \mathrm{~mm}$, respectively; $P<$ 0.001 ), followed by those chicks switched from 500 to 40,000 $\operatorname{lux}(\Delta+0.64 \pm 0.13$ vs. $\Delta+0.39 \pm 0.03 \mathrm{~mm}$, respectively; $P<$ 0.001 ; Table 6).

\section{Exposure to Bright Light Over a 7-Day Period Does Not Induce Retinal Damage}

Retinal morphology was unaffected by 7 days of exposure to bright light (40,000 lux, Fig. 4A) relative to that seen in animals reared under 500 lux (Fig. 4B). For TUNEL staining, incubation with secondary antibody alone (negative control) 
TABLE 4. Axial Length Data for Age-Matched Untreated Control Eyes Over 11 Days for All Light Intensities

\begin{tabular}{ccccc}
\hline & & \multicolumn{3}{c}{ Axial Length, mm } \\
\cline { 3 - 5 } Treatment & LI, Lux & Day 0 & Day 6 & Day 9 \\
\hline Untreated control & 40,000 & $8.61 \pm 0.08$ & $8.65 \pm 0.05$ & $8.77 \pm 0.06$ \\
Untreated control & 30,000 & $8.58 \pm 0.03$ & $8.74 \pm 0.03$ & \\
Untreated control & 20,000 & $8.56 \pm 0.04$ & $8.81 \pm 0.04$ & \\
Untreated control & 10,000 & $8.58 \pm 0.05$ & $8.63 \pm 0.03$ & 0.05 \\
Untreated control & 500 & $8.50 \pm 0.06$ & $8.82 \pm 0.06$ \\
\hline
\end{tabular}

showed no specific cell labeling (Fig. 4C). In contrast, positive control sections from light-damaged retinal tissue, induced by 24 hours of exposure to 1500 lux in SD rats, showed significant TUNEL staining of photoreceptor cell bodies in the outer nuclear layer (Fig. 4D). TUNEL staining was not observed in retinal tissue from either bright light-treated (40,000 lux, Fig. $4 \mathrm{E})$ or normal light-reared animals (500 lux, Fig. 4F). This suggests that daily exposure to 40,000 lux over a period of 7 days does not induce retinal cell apoptosis in chicks.

\section{Discussion}

A strong negative correlation was observed between the development of deprivation myopia and the intensity of light to which the animals were exposed, with greater protection provided with higher light intensities. This negative correlation was best described by a logarithmic relationship. With 40,000 lux, the development of FDM was almost completely abolished when compared to the refractive changes observed in both contralateral control eyes and age-matched untreated animals. Consistent with the changes observed in refraction, the excessive axial elongation associated with deprivation myopia was retarded, to increasing degrees, relative to the light intensity to which animals were exposed. With 40,000 lux, changes in axial length were not statistically different from contralateral control or age-matched untreated values.

\section{What Are the Relevant Light Intensities Required to Prevent Deprivation Myopia?}

In rhesus monkeys, daily exposure to 18,000 to 28,000 lux for 6 hours per day abolished the development of FDM and in fact induced a small hyperopic shift. ${ }^{16}$ Similar light levels in chicks (20,000 and 30,000 lux) retard the development of FDM by roughly $70 \%$ and $80 \%$, respectively, with near-complete protection $(\sim 95 \%)$ achieved at higher light levels $(40,000$ lux). This difference between the rhesus monkey and chicken is likely due to slight variations in the experimental protocols. For example, differences in the diffuser construct may lead to differences in the degree to which light is attenuated.

Similar to chicks, tree shrews show a roughly $60 \%$ reduction in FDM in response to 15,000 lux. ${ }^{17}$ This may suggest that FDM would also be abolished in tree shrews by illumination levels in the range of 40,000 lux. Interestingly, in both tree shrews ${ }^{17}$ and rhesus monkeys, ${ }^{16}$ a relative hyperopic shift in refraction is seen in contralateral control and age-matched untreated eyes in response to high light. A similar hyperopic shift was not

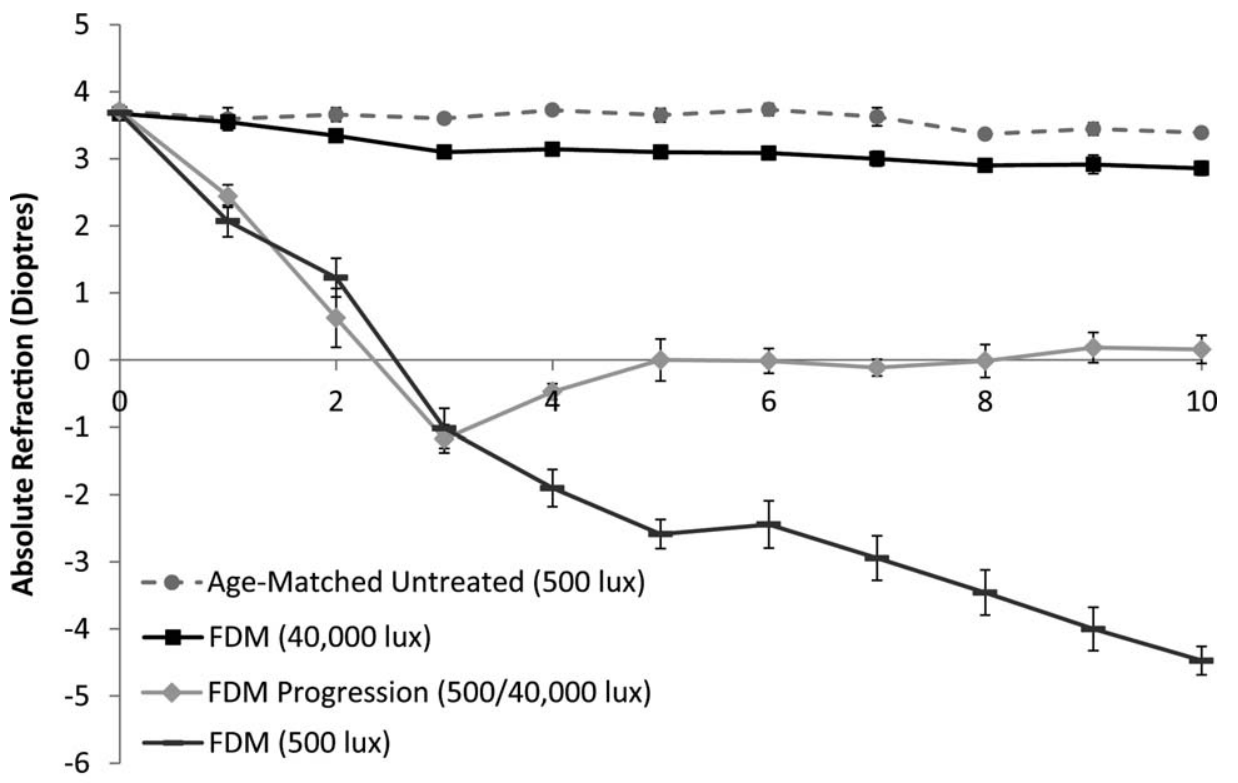

Time (days)

Figure 3. Absolute change in refraction, over 11 days, for diffuser-treated eyes from animals reared under one of three lighting conditions (500, 40,000 , or 500 lux for 3 days followed by 40,000 lux for 8 days). Further progression of FDM was halted once chicks were switched from low light (500 lux) to high light (40,000 lux), relative to those chicks reared continuously under 500 lux. Plotted untreated control values represent the mean refraction at each time point for age-matched untreated control eyes. There was no statistical difference in refraction changes seen in contralateral control eyes or age-matched untreated control eyes in response to any light intensity. Therefore contralateral data are not plotted for ease of viewing. Error bars: standard error of the mean, $n=12$ per group. 
observed in chicks; instead, refractive changes in both contralateral control and age-matched untreated eyes were relatively stable, with a mild reduction in the hyperopic values seen over time. This lack of a relative hyperopic shift may be due to the shorter time course investigated in chicks. However, Cohen et al. ${ }^{20}$ did not observe a relative hyperopic shift in chicks reared under high light for 90 days, supporting the idea that this phenomenon may not be observed in chicks. It should be noted, though, that 10,000 lux was the maximum illumination level used.

While the current experiments have shown that almost complete inhibition of the development of FDM can be achieved by increasing the light intensity, it is still not known what the optimal duration of high light exposure is. Also, we do not yet know if there is an interaction between duration and intensity, such that short periods of exposure to more intense illumination would produce protective effects similar to those of longer periods under less intense lighting. Work in chicks has indicated that doubling the exposure time to high light $(15,000$ lux ) from 5 hours per day to 10 hours per day does not increase the retardation of FDM. ${ }^{23}$ However, providing short pulses of high light over a 5-hour period per day, rather than one continuous burst of high light over the same period of time ( 5 hours per day), appears to enhance the protective effect. ${ }^{23}$ This may suggest that both intensity and timing are of importance in maximizing the protective effects of light.

\section{The Effect of High Light on Lens Compensation}

Exposure to elevated light levels also affects the rate of compensation to imposed optical defocus. In chicks and tree shrews, daily exposure to high light significantly reduces the rate of compensation to negative lenses, although compensation is still achieved. ${ }^{15,17}$ In contrast, exposure to high light appears not to affect the rate of compensation to negative lenses in rhesus monkeys. ${ }^{24}$ This result is somewhat surprising, as the ability of light to retard the development of FDM appears to be highly conserved between chicks, tree shrews, and rhesus monkeys. The effect of high light on plus-lens compensation has been analyzed only in chicks. Here, daily exposure to 15,000 lux enhanced the "STOP" growth signal already induced by plus-lenswear, increasing the rate of compensation to the imposed myopic defocus. ${ }^{15}$

\section{Can Myopia Progression Be Halted by Exposure to Elevated Light Intensities?}

Although high light is capable of retarding the onset of FDM, a crucial question, especially for human application, is whether exposure to high light can prevent further progression in already myopic animals. Recent epidemiological findings have indicated that increased time outdoors can prevent the onset of myopia, but not the rate of progression. ${ }^{12,13}$ We therefore wished to determine whether high light exposure can prevent the excessive axial elongation that underlies both the onset and progression of myopia. Promisingly, the progress of FDM was halted on switching animals from a 500-lux environment to daily exposure to 40,000 lux for a period of 6 hours per day. Importantly, this protective effect was associated with a reduced rate of axial elongation. An initial relative hyperopic shift was observed during the first 2 days post switch, before refractive values plateaued around emmetropia. Based on the size and rapid rate of the initial relative hyperopic shift, this most likely represented a choroidal response (choroidal expansion), which is commonly observed in chicks during periods of reduced ocular growth associated with recovery from experimental myopia or plus-lenswear. ${ }^{25,26}$ The ability of bright light to halt the progression of deprivation myopia is a 
Table 6. Axial Length Data From Diffuser-Treated Eyes Over 10 Days for Chicks Exposed to 500, 40,000, or 500 Lux for 3 Days, Followed by 40,000 Lux for 8 Days

\begin{tabular}{lcrr}
\hline & & & Axial Length, mm \\
\cline { 3 - 4 } \multicolumn{1}{c}{ Treatment } & LI, Lux & Day 0 & Day 9 \\
\hline Constant diffuser & 40,000 & $8.58 \pm 0.07$ & $9.05 \pm 0.15$ \\
Contralateral control & 40,000 & $8.58 \pm 0.07$ & $8.89 \pm 0.09$ \\
Relative change in axial length & & $0.00 \pm 0.14$ & $+0.17 \pm 0.10$ \\
Constant diffuser & $500 / 40,000$ & $8.61 \pm 0.07$ & $9.35 \pm 0.13$ \\
Contralateral control & $500 / 40,000$ & $8.58 \pm 0.07$ & $8.97 \pm 0.12$ \\
Relative change in axial length & & $+0.03 \pm 0.08$ & $+0.39 \pm 0.03$ \\
Constant diffuser & 500 & $8.55 \pm 0.08$ & $9.54 \pm 0.13$ \\
Contralateral control & 500 & $8.58 \pm 0.07$ & $8.90 \pm 0.04$ \\
Relative change in axial length & & $-0.03 \pm 0.09$ & $+0.64 \pm 0.13$ \\
\hline
\end{tabular}
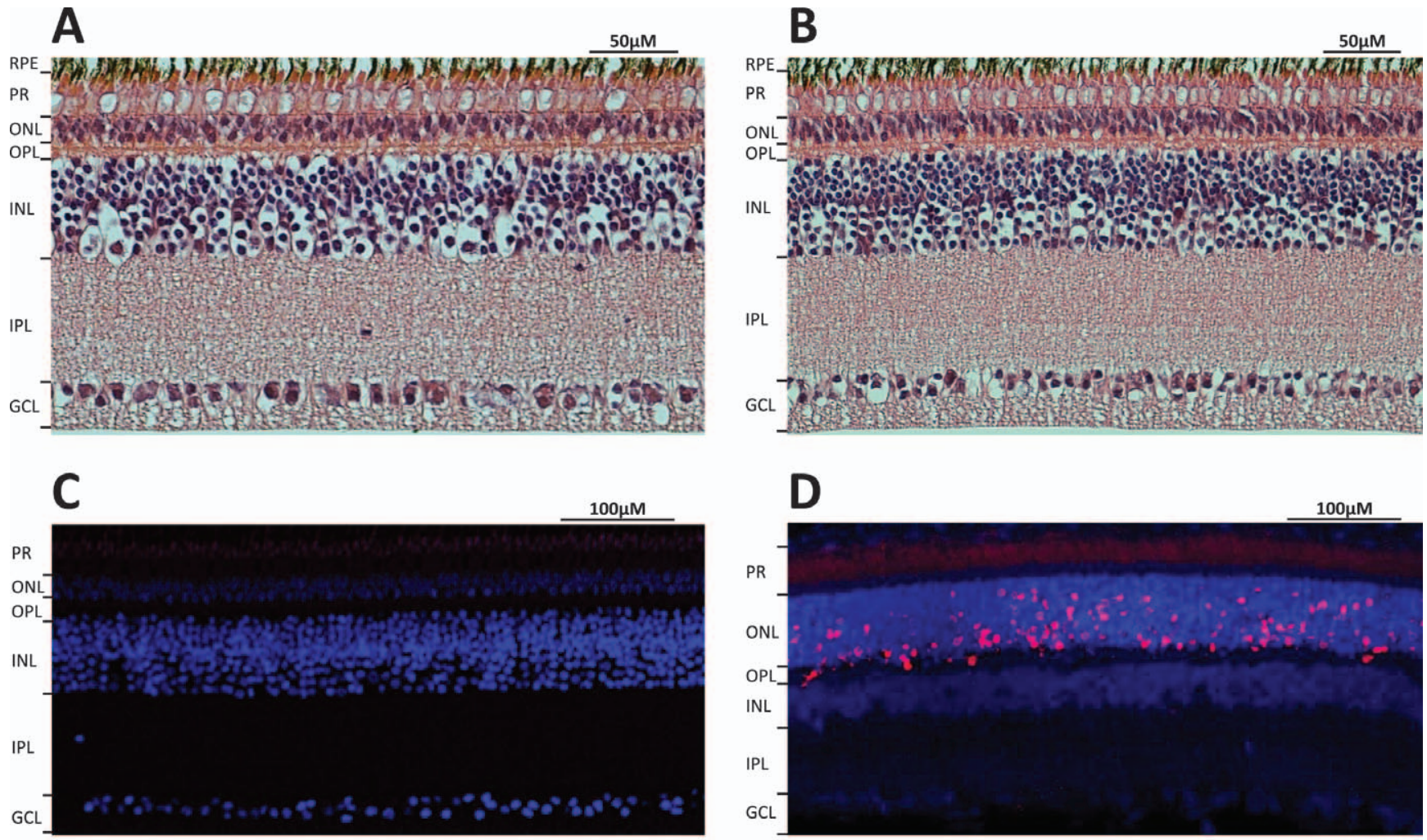

\section{E}
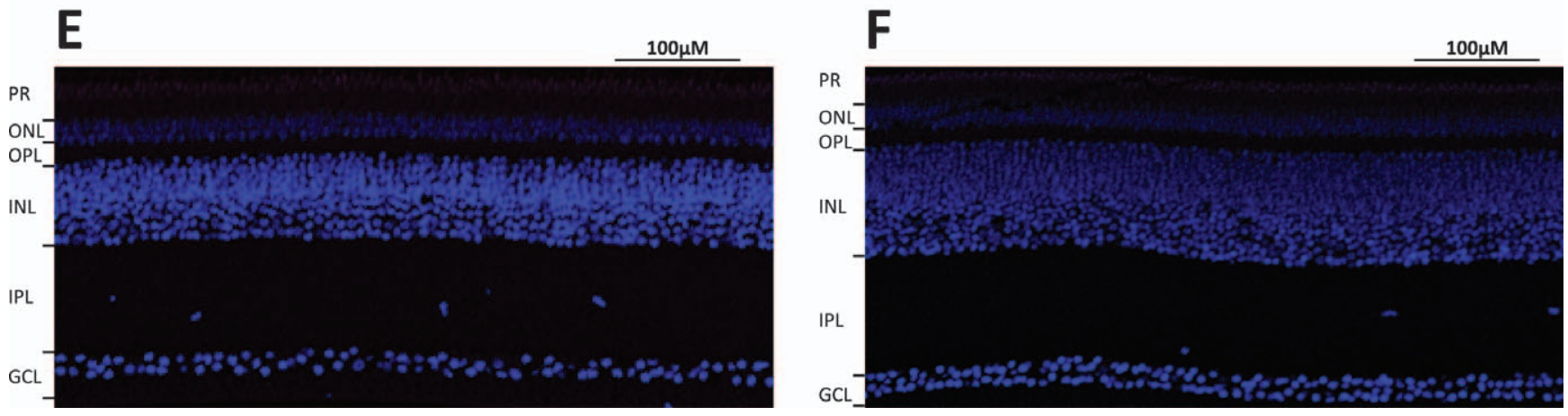

GCL

Figure 4. Histology of retinal tissue from bright light-treated chicks. (A, B) Retinal sections stained with H\&E from bright light (40,000 lux [A])and normal light (500 lux [B])-treated chicks. No differences in general retinal histology were detectable between the two light treatments. (C-F) TUNEL-labeled sections, costained with DAPI. (C) Incubation with secondary antibody alone (negative control) did not show specific cell labeling, with only weak autofluorescence observed. (D) Positive TUNEL control tissue. DNA damage and apoptosis was observed in photoreceptor cell bodies located in the outer nuclear layer of the SD rat retina following light damage induced by exposure to 1500 lux for a period of 24 hours. This tissue was processed in parallel with retinal tissue obtained from the chicken. (E, F) Retinal sections from bright light (E)- and normal light (F)treated birds. Neither lighting condition showed TUNEL labeling. 
promising finding, especially if translatable to human populations, where stopping further axial elongation can prevent the development of chorioretinal pathologies.

\section{Possible Mechanisms Underlying the Protective Effects of High Light Exposure}

A number of possible factors have been proposed to explain the protective effects of bright light (for review see Refs. 14, 15, 27). For example, bright light will lead to pupil constriction and hence greater depth of focus, bringing about reduced image blur. However, this theory does not explain the ability of high light to retard the development of FDM, as pupil constriction will be unable to alter the image blur associated with the attached diffusers. Increased optical flow rates, previously shown to retard the development of FDM, ${ }^{28}$ could be associated with greater movement/activity under the higher lights. However, chickens were found not to change their rate of activity (movement) under high lights. ${ }^{14}$ A role for UV light and vitamin D levels has also been proposed, with two recent studies reporting that vitamin D levels appear to be slightly lower in myopes than nonmyopes, ${ }^{29,30}$ while an association between vitamin $\mathrm{D}$ receptor polymorphisms and myopia has also been observed. ${ }^{31}$ However, the lighting systems used to retard the development of experimental myopia in animal models do not produce light in the UV spectrum; therefore UV exposure cannot explain this protective effect. ${ }^{14-17,24}$ Further, there is no significant difference in the compensation response of chicks to optical defocus under either white light or bright UV light, ${ }^{32}$ suggesting that UV exposure does not modify the emmetropization response. In terms of vitamin $\mathrm{D}$ levels, vitamin $\mathrm{D}_{3}$ supplementation does not affect the development of FDM or lens-induced myopia (LIM) in the tree shrew (Siegwart JT, et al. IOVS 2011;52:ARVO E-Abstract 6298), and therefore this study does not support a role for vitamin $\mathrm{D}$ in the development of experimental myopia.

Instead, as hypothesized by Rose and colleagues, ${ }^{10}$ evidence from animal studies suggests that the protective effects afforded by bright light are driven by increased retinal dopamine levels. Four points of evidence support a role for dopamine in the protective effects of bright light. Firstly, the release of dopamine, a light-driven neurohormone, from amacrine and/or interplexiform cells increases in a log-linear fashion over the light ranges normally seen during the day (100-100,000 lux). ${ }^{33,34}$ Secondly, retinal dopamine levels are reduced during the development of experimental myopia (for review see Ref. 35), with dopamine D2 receptor agonists capable of retarding the development of experimental myopia. ${ }^{36-40}$ Thirdly, the ability of brief periods of normal vision (diffuser removal) to retard the development of FDM, ${ }^{18,19}$ a process that is enhanced under bright light, ${ }^{14}$ is abolished by the administration of the dopamine D2 receptor antagonist spiperone. ${ }^{39}$ Finally, the protective effects afforded by bright light against the development of deprivation myopia in chicks is abolished by daily injection of spiperone. ${ }^{15}$

The current findings further illustrate that altering the lighting conditions under which animals are reared can significantly affect ocular development. For example, circadian and/or diurnal entrainment is critical to normal ocular development (for review see Ref. 41). Disruption to such rhythmicity, by rearing of chicks in either constant light ${ }^{42-49}$ or constant dark, ${ }^{47,50,51}$ produces vitreal chamber enlargement, thickening and flattening of the cornea, and shallowing of the anterior chamber, with an overall hyperopic shift in refraction. Chromatic cues also appear capable of influencing normal ocular development (for review see Ref. 52). Specifically, rearing animals in monochromatic red light, in which the focal plane falls behind the retina, induces a relative myopic shift in refraction (increased axial elongation) compared to values in animals reared in monochromatic blue light, in which the focal plane falls in front of the retina. ${ }^{53-57}$ Finally, as demonstrated here, light levels can significantly affect normal ocular growth ${ }^{20,58-60}$ or the response of the eye to optical manipulation. ${ }^{14-17}$

\section{The Role of Light Intensity in Human Myopia}

Data from clinical trials in Taiwan ${ }^{61}$ and preliminary data from trials in China (Morgan IG, et al. IOVS 2014;55:ARVO E-Abstract 1272), associated with increasing the time spent outdoors by children during the school day, have shown promising initial results. If findings from experimental models of myopia are directly translatable to human myopia, work in the rhesus monkey, which is phylogenetically the closest of the models to humans, indicates that daily exposure to light intensities in the range of 18,000 to 28,000 lux could block the onset of myopia in children. Further, the current findings suggest that progression in already myopic eyes can be halted by light levels similar to those required to block onset. Such light intensities are easily achievable outdoors, with luminance levels ranging from 20,000 to 30,000 lux on a cloudy day or in the shade, to in excess of 100,000 lux on a bright sunny day.

In summary, the level of protection from the development of FDM increased with increasing light intensity. Exposure to 40,000 lux for a period of 6 hours per day provided nearcomplete protection against the onset of deprivation myopia while halting any further progression in already myopic eyes. Importantly, high light exposure prevented the excessive axial elongation that underlies both the onset and progression of FDM.

\section{Acknowledgments}

Supported by funding from the Centre for Research into Therapeutic Solutions at the University of Canberra, Australian Capital Territory, Australia. The authors thank Riccardo Natoli and Kartik Saxena for their help with the histology presented.

Disclosure: C. Karouta, None; R.S. Ashby, None

\section{References}

1. Matsumura $\mathrm{H}$, Hirai $\mathrm{H}$. Prevalence of myopia and refractive changes in students from 3 to 17 years of age. Surv Ophthalmol. 1999;44(suppl 1):S109-S115.

2. Wu HM, Seet B, Yap EP, Saw SM, Lim TH, Chia KS. Does education explain ethnic differences in myopia prevalence? A population-based study of young adult males in Singapore. Optom Vis Sci. 2001;78:234-239.

3. Lin LL, Shih YF, Hsiao CK, Chen CJ. Prevalence of myopia in Taiwanese schoolchildren: 1983 to 2000. Ann Acad Med Singapore. 2004;33:27-33.

4. Morgan IG, Ohno-Matsui K, Saw SM. Myopia. Lancet. 2012; 379:1739-1748.

5. Goh WS, Lam CS. Changes in refractive trends and optical components of Hong Kong Chinese aged 19-39 years. Ophthalmic Physiol Opt. 1994;14:378-382.

6. Jung SK, Lee JH, Kakizaki H, Jee D. Prevalence of myopia and its association with body stature and educational level in 19year-old male conscripts in Seoul, South Korea. Invest Ophthalmol Vis Sci. 2012;53:5579-5583.

7. Dirani M, Tong L, Gazzard G, et al. Outdoor activity and myopia in Singapore teenage children. Br J Ophthalmol. 2009; 93:997-1000. 
8. Jones LA, Sinnott LT, Mutti DO, Mitchell GL, Moeschberger ML, Zadnik K. Parental history of myopia, sports and outdoor activities, and future myopia. Invest Ophthalmol Vis Sci. 2007; 48:3524-3532.

9. Mutti DO, Mitchell GL, Moeschberger ML, Jones LA, Zadnik K. Parental myopia, near work, school achievement, and children's refractive error. Invest Ophthalmol Vis Sci. 2002;43: 3633-3640.

10. Rose KA, Morgan IG, Ip J, et al. Outdoor activity reduces the prevalence of myopia in children. Opbthalmology. 2008;115: 1279-1285.

11. Rose KA, Morgan IG, Smith W, Burlutsky G, Mitchell P, Saw SM. Myopia, lifestyle, and schooling in students of Chinese ethnicity in Singapore and Sydney. Arch Ophthalmol. 2008; 126:527-530.

12. Jones-Jordan LA, Sinnott LT, Cotter SA, et al. Time outdoors, visual activity, and myopia progression in juvenile-onset myopes. Invest Ophthalmol Vis Sci. 2012;53:7169-7175.

13. French AN, Morgan IG, Mitchell P, Rose KA. Risk factors for incident myopia in Australian schoolchildren: the Sydney adolescent vascular and eye study. Ophthalmology. 2013;120: 2100-2108.

14. Ashby R, Ohlendorf A, Schaeffel F. The effect of ambient illuminance on the development of deprivation myopia in chicks. Invest Ophthalmol Vis Sci. 2009;50:5348-5354.

15. Ashby RS, Schaeffel F. The effect of bright light on lens compensation in chicks. Invest Ophthalmol Vis Sci. 2010;51: 5247-5253.

16. Smith EL III, Hung LF, Huang J. Protective effects of high ambient lighting on the development of form-deprivation myopia in rhesus monkeys. Invest Ophthalmol Vis Sci. 2012; 53:421-428.

17. Norton TT, Siegwart JT Jr. Light levels, refractive development, and myopia-a speculative review. Exp Eye Res. 2013;114:4857.

18. Napper GA, Brennan NA, Barrington M, Squires MA, Vessey GA, Vingrys AJ. The duration of normal visual exposure necessary to prevent form deprivation myopia in chicks. Vision Res. 1995;35:1337-1344.

19. Napper GA, Brennan NA, Barrington M, Squires MA, Vessey GA, Vingrys AJ. The effect of an interrupted daily period of normal visual stimulation on form deprivation myopia in chicks. Vision Res. 1997;37:1557-1564.

20. Cohen Y, Belkin M, Yehezkel O, Solomon AS, Polat U. Dependency between light intensity and refractive development under light-dark cycles. Exp Eye Res. 2011;92:40-46.

21. Maslim J, Valter K, Egensperger R, Hollander H, Stone J. Tissue oxygen during a critical developmental period controls the death and survival of photoreceptors. Invest Ophthalmol Vis Sci. 1997;38:1667-1677.

22. Rutar M, Provis JM, Valter K. Brief exposure to damaging light causes focal recruitment of macrophages, and long-term destabilization of photoreceptors in the albino rat retina. Curr Eye Res. 2010;35:631-643.

23. Lan W, Feldkaemper M, Schaeffel F. Intermittent episodes of bright light suppress myopia in the chicken more than continuous bright light. PLoS One. 2014;9:e110906.

24. Smith EL III, Hung LF, Arumugam B, Huang J. Negative lensinduced myopia in infant monkeys: effects of high ambient lighting. Invest Ophthalmol Vis Sci. 2013;54:2959-2969.

25. Wallman J, Wildsoet $\mathrm{CF}, \mathrm{Xu} \mathrm{A}$, et al. Moving the retina: choroidal modulation of refractive state. Vision Res. 1995;35: 37-50.

26. Wildsoet C, Wallman J. Choroidal and scleral mechanisms of compensation for spectacle lenses in chicks. Vision Res. 1995; 35:1175-1194.
27. French AN, Ashby RS, Morgan IG, Rose KA. Time outdoors and the prevention of myopia. Exp Eye Res. 2013;114:58-68.

28. Schwahn HN, Schaeffel F. Flicker parameters are different for suppression of myopia and hyperopia. Vision Res. 1997;37: 2661-2673.

29. Choi JA, Han K, Park YM, La TY. Low serum 25-hydroxyvitamin $\mathrm{D}$ is associated with myopia in Korean adolescents. Invest Ophthalmol Vis Sci. 2014;55:2041-2047.

30. Yazar S, Hewitt AW, Black LJ, et al. Myopia is associated with lower vitamin D status in young adults. Invest Ophthalmol Vis Sci. 2014;55:4552-4559.

31. Mutti DO, Cooper ME, Dragan E, et al. Vitamin D receptor (VDR) and group-specific component (GC, vitamin D-binding protein) polymorphisms in myopia. Invest Ophthalmol Vis Sci. 2011;52:3818-3824.

32. Hammond DS, Wildsoet CF. Compensation to positive as well as negative lenses can occur in chicks reared in bright UV lighting. Vision Res. 2012;67:44-50.

33. Cohen Y, Peleg E, Belkin M, Polat U, Solomon AS. Ambient illuminance, retinal dopamine release and refractive development in chicks. Exp Eye Res. 2012;103:33-40.

34. Morgan IG, Boelen MK. Complexity of dopaminergic function in the retinal dark-light switch. Aust N Z J Opbthalmol. 1996; 24:56-58.

35. Feldkaemper M, Schaeffel F. An updated view on the role of dopamine in myopia. Exp Eye Res. 2013;114:106-119.

36. Stone RA, Lin T, Laties AM, Iuvone PM. Retinal dopamine and form-deprivation myopia. Proc Natl Acad Sci U S A. 1989;86: 704-706.

37. Iuvone PM, Tigges M, Stone RA, Lambert S, Laties AM. Effects of apomorphine, a dopamine receptor agonist, on ocular refraction and axial elongation in a primate model of myopia. Invest Ophthalmol Vis Sci. 1991;32:1674-1677.

38. Ashby R, McCarthy CS, Maleszka R, Megaw P, Morgan IG. A muscarinic cholinergic antagonist and a dopamine agonist rapidly increase ZENK mRNA expression in the form-deprived chicken retina. Exp Eye Res. 2007;85:15-22.

39. McCarthy CS, Megaw P, Devadas M, Morgan IG. Dopaminergic agents affect the ability of brief periods of normal vision to prevent form-deprivation myopia. Exp Eye Res. 2007;84:100107.

40. Mao J, Liu S, Qin W, Li F, Wu X, Tan Q. Levodopa inhibits the development of form-deprivation myopia in guinea pigs. Optom Vis Sci. 2010;87:53-60.

41. Nickla DL. Ocular diurnal rhythms and eye growth regulation: where we are 50 years after Lauber. Exp Eye Res. 2013;114: 25-34.

42. Cohen Y, Belkin M, Yehezkel O, Avni I, Polat U. Light intensity modulates corneal power and refraction in the chick eye exposed to continuous light. Vision Res. 2008;48:2329-2335.

43. Li T, Howland HC. Modulation of constant light effects on the eye by ciliary ganglionectomy and optic nerve section. Vision Res. 2000;40:2249-2256.

44. Li T, Howland HC. The effects of constant and diurnal illumination of the pineal gland and the eyes on ocular growth in chicks. Invest Ophthalmol Vis Sci. 2003;44:36923697.

45. Li T, Howland HC, Troilo D. Diurnal illumination patterns affect the development of the chick eye. Vision Res. 2000;40: 2387-2393.

46. Li T, Troilo D, Glasser A, Howland HC. Constant light produces severe corneal flattening and hyperopia in chickens. Vision Res. 1995;35:1203-1209.

47. Osol G, Schwartz B, Foss DC. The effects of photoperiod and lid suture on eye growth in chickens. Invest Ophthalmol Vis Sci. 1986;27:255-260. 
48. Stone RA, Lin T, Desai D, Capehart C. Photoperiod, early postnatal eye growth, and visual deprivation. Vision Res. 1995;35: 1195-1202.

49. Lauber JK, Oishi T. Lid suture myopia in chicks. Invest Ophthalmol Vis Sci. 1987;28:1851-1858.

50. Yinon U, Koslowe KC. Eyelid closure effects on the refractive error of the eye in dark- and in light-reared kittens. $A m J$ Optom Physiol Opt. 1984;61:271-273.

51. Yinon U, Koslowe KC, Rassin MI. The optical effects of eyelid closure on the eyes of kittens reared in light and dark. Curr Eye Res. 1984;3:431-439.

52. Rucker FJ. The role of luminance and chromatic cues in emmetropisation. Ophthalmic Physiol Opt. 2013;33:196-214.

53. Long Q, Chen D, Chu R. Illumination with monochromatic long-wavelength light promotes myopic shift and ocular elongation in newborn pigmented guinea pigs. Cutan Ocul Toxicol. 2009;28:176-180.

54. Rohrer B, Schaeffel F, Zrenner E. Longitudinal chromatic aberration and emmetropization: results from the chicken eye. J Physiol. 1992;449:363-376.
55. Seidemann A, Schaeffel F. Effects of longitudinal chromatic aberration on accommodation and emmetropization. Vision Res. 2002;42:2409-2417.

56. Liu R, Qian YF, He JC, et al. Effects of different monochromatic lights on refractive development and eye growth in guinea pigs. Exp Eye Res. 2011;92:447-453.

57. Rucker FJ, Wallman J. Chick eyes compensate for chromatic simulations of hyperopic and myopic defocus: evidence that the eye uses longitudinal chromatic aberration to guide eyegrowth. Vision Res. 2009; 49:1775-1783.

58. Gottlieb MD, Fugate-Wentzek LA, Wallman J. Different visual deprivations produce different ametropias and different eye shapes. Invest Ophthalmol Vis Sci. 1987;28:1225-1235.

59. Lauber JK, Kinnear A. Eye enlargement in birds induced by dim light. Can J Opbthalmol. 1979;14:265-269.

60. Feldkaemper M, Diether S, Kleine G, Schaeffel F. Interactions of spatial and luminance information in the retina of chickens during myopia development. Exp Eye Res. 1999;68:105-115.

61. Wu PC, Tsai CL, Wu HL, Yang YH, Kuo HK. Outdoor activity during class recess reduces myopia onset and progression in school children. Opbthalmology. 2013;120:1080-1085. 